\title{
La disparition de l'expertise (en médecine)
}

Les opinions exprimées dans cet éditorial sont celles de l'auteur et ne représentent pas nécessairement celles de l'Association médicale canadienne ou ses filiales.

'expertise. En tant que médecins et chirurgiens, nous devrions tous posséder cette qualité. C'est une notion que j'ai toujours tenue pour acquise. Mais il semble que la société ne soit pas du même avis. Le concept a du bon et du moins bon. Il ne semble plus nécessaire de posséder une expertise pour réussir dans le monde actuel - même pour diriger un pays. De nos jours, les gens atteignent des positions de pouvoir en ne se souciant que du résultat final, et certainement sans la formation nécessaire pour être compétents. Je crois qu'il est bon de faire preuve d'une certaine transparence en médecine. Il peut être fatigant de se faire poser des questions sur des renseignements trouvés sur Google, mais le fait que les patients s'informent donne lieu à un véritable dialogue.

Le problème, c'est que des non-scientifiques qui se basent sur des recherches Google tuent des gens avec leurs fables médicales. Pensons entre autres à la rhétorique anti-vaccination. On trouve de plus en plus de sites Web qui paraissent sérieux, mais qui contiennent des renseignements pour le moins étranges. Ces sites prétendent fournir de l'information que les « géants de la médecine » cherchent à garder secrète et donnent au lecteur l'impression que nous, le monde médical, lui cachons des choses - de la même manière que Trump a mené sa campagne. Cela dégrade la relation médecinpatient. On lit dans des sources aux titres officiels que des traitements médicaux tuent plus de gens que les maladies elles-mêmes, et que les médecins n'offrent ces traitements que pour s'enrichir. Ce sont là des fausses nouvelles à l'état pur, présentées comme «preuves » que la médecine occidentale ne fonctionne pas.

Alors, comment la médecine est-elle touchée par la perte d'importance de l'expertise? Chaque semaine, comme bien d'autres universitaires, je reçois des dizaines d'invitations à siéger aux comités de révision ou de rédaction de toutes nouvelles revues. Il s'agit généralement de revues en accès libre qui contiennent des titres parfois intéressants et presque exacts, mais sont publiées par des éditeurs rapaces. Quand l'avalanche d'offres a commencé, j'ai eu envie de participer; heureusement, ma réputation n'a pas été ternie. Mais le déferlement constant de nouveaux titres n'est pas sans rappeler la désinformation sur Internet mentionnée plus haut. La dilution du contrôle de quelques maisons d'édition n'est pas une mauvaise chose en soi, mais la dilution de l'expertise dans les comités de révision, et la baisse de la qualité des manuscrits qui s'ensuit sont plutôt inquiétantes. Peut-être avons-nous atteint le vrai modèle d'Internet consistant à « tout publier et laisser le lecteur faire le tri »? Si oui, je ne crois pas que le lecteur soit au courant. La plupart des gens qui lisent des revues scientifiques croient encore que les articles sont approuvés et évalués par les pairs, et que leur validité est vérifiée. Pourtant, même avec l'ancien système, ces 3 principes étaient difficiles à garantir.

Par conséquent, l'accès libre est-il un mauvais mécanisme de diffusion? Bien sûr que non. Faut-il éviter à tout prix les nouvelles revues? Pas du tout. Gardons toutefois en tête qu'une qualité demeure essentielle en médecine, autant pour les lecteurs d'articles scientifiques que pour les équipes d'édition et de révision qui les publient. L'expertise.

\section{Edward J. Harvey, MD \\ Corédacteur, Fournal canadien de chirurgie}

Intérêts concurrents: E.J. Harvey est médecin hygiéniste en chef de Greybox Healthcare (Montréal) et président du Conseil d'administration de NXT-Sens Inc. (Montréal).

DOI: $10.1503 /$ cjs.001218 